\title{
New perspective in degradation mechanism of $\mathrm{SrTiO}_{3}: \mathrm{Pr}, \mathrm{Al}, \mathrm{Ga}$ phosphors
}

\author{
Jin Young Kim, ${ }^{\text {a) }}$ Yong Chan You, Jong Hyuk Kang, and Duk Young Jeon \\ Department of Materials Science and Engineering, Korea Advanced Institute of Science and \\ Technology (KAIST), Yusung-gu, Daejeon 305-701, Korea \\ Jörg Weber \\ Institute for Applied Physics-Semiconductor Physics, TUI-Dresden, Dresden D-01062, Germany
}

(Received 3 February 2004; accepted 2 June 2004)

\begin{abstract}
Under prolonged electron-beam exposure, perovskite-structured $\mathrm{SrTiO}_{3}: \mathrm{Pr}, \mathrm{Al}, \mathrm{Ga}(\mathrm{STO})$ phosphor can be easily reduced due to oxygen loss. In particular, it is well known that dissociative $\mathrm{H}_{2} \mathrm{O}$ molecules are well adsorbed on reduced STO surfaces. The hydroxyl species produced by such dissociative adsorption of $\mathrm{H}_{2} \mathrm{O}$ strongly decompose organic compounds chemisorbed on the surface from vacuum ambient used in display devices into carbon species due to the photocatalytic properties of STO. Consequently, it is very likely that this mechanism attributes to the larger amounts of carbon adsorption by electron-stimulated chemical reactions on the STO phosphor surface than other phosphors.
\end{abstract}

\section{INTRODUCTION}

Recently, vacuum fluorescent displays (VFDs) have been investigated for application as display devices for dashboards in cars and cockpits in airplanes, placing severe demands for screen brightness. ${ }^{1}$ Above all, under these applications, to maintain the adjusted initial color coordinate and such high brightness, the phosphor particles are irradiated for a long time under a high anode current. If any of the selected red, green, and blue (RGB) phosphors tailored for the VFDs suffer from this environment, the light output will decrease, resulting in a color coordinate shift in the display over its lifetime. ${ }^{2}$ Thus for applications where high current density of electron beam is used, such as in VFDs, the degradation behavior is expected to be a major selection criterion for the phosphor. ${ }^{3}$ Satisfying these requirements, VFD manufacturers have selected phosphors that are already known and reported in literature. ${ }^{4}$ However, among the RGB phosphors for the VFDs, $(\mathrm{Zn}, \mathrm{Cd}) \mathrm{S}$ : Ag, $\mathrm{Cl}$ currently used as the red-emitting phosphor needs to be substituted by other oxide phosphors in the near future due to instability of the host lattice, such as sulfide and Cd-containing material. ${ }^{5-7}$ Thus, the development of new red-emitting phosphors with high efficiency is urgently needed for low voltage display applications. In 1996, a new red-emitting phosphor, $\mathrm{SrTiO}_{3}: \mathrm{Pr}^{3+}$ was successfully developed by adding $\mathrm{Al}$ and/or $\mathrm{Ga}$, which offers the high low voltage cathodoluminescence (CL) efficiency

\footnotetext{
a) Address all correspondence to this author.

e-mail: tg2som@naver.com

DOI: $10.1557 / J M R .2004 .0337$
}

and excellent color purity. ${ }^{8-10} \mathrm{SrTiO}_{3}: \mathrm{Pr}, \mathrm{Al}, \mathrm{Ga}$ (STO) phosphor-based low voltage display devices, however, have exhibited short lifetimes. The characteristics of these drawbacks include rapid degradation of low voltage CL of phosphors. Even though there have been several reports on these properties, there is no clear-cut understanding yet. ${ }^{11-13}$ Thus, in this study, we propose a new mechanism which can provide explict understanding as to the rapid degradation of low voltage CL.

\section{EXPERIMENTAL}

\section{A. Synthesis of phosphors}

The $\mathrm{SrTiO}_{3}: \mathrm{Pr}, \mathrm{Al}, \mathrm{Ga}$ phosphor samples were prepared with solid-state reaction among $\mathrm{Sr}\left(\mathrm{NO}_{3}\right)_{2}, \mathrm{TiO}_{2}, \mathrm{PrCl}_{3}$, $\mathrm{Al}(\mathrm{OH})_{3}$, and $\mathrm{Ga}_{2} \mathrm{O}_{3}$. The phosphor powders were prepared in a series of mixing, calcining, ball-milling, sieving steps. The calcining is done in air at $1300{ }^{\circ} \mathrm{C}$ for $4 \mathrm{~h}$. Figure 1 shows the $\mathrm{x}$-ray diffraction (XRD) patterns of $\mathrm{SrTiO}_{3}: \mathrm{Pr}^{3+}(0.2 \mathrm{~mol} \%)$ doped with $20 \mathrm{~mol} \%$ of $\mathrm{Al}^{3+}$ and/or $\mathrm{Ga}^{3+}$. As a reference, an XRD pattern of $\mathrm{SrTiO}_{3}$ is also shown in Fig. 1. A change is not observed in the XRD pattern by the addition of group-III $b$ ions. The CL spectrum of $\mathrm{SrTiO}_{3}: \mathrm{Pr}, \mathrm{Al}, \mathrm{Ga}$ is shown in Fig. 2. The CL spectrum has a peak at $617 \mathrm{~nm}$, which is attributed to the intra- $4 \mathrm{f}$ transitions from the excited state ${ }^{1} \mathrm{D}_{2}$ to the ground state ${ }^{3} \mathrm{H}_{4}$ of $\operatorname{Pr}^{3+}\left({ }^{1} \mathrm{D}_{2} \rightarrow{ }^{3} \mathrm{H}_{4} \mathrm{CL}\right) .{ }^{9}$

\section{B. Preparation of degraded samples and luminescence measurements by the low voltage electron irradiation}

The synthesized phosphor was molded into a pellet of a disk and placed inside a vacuum chamber that 


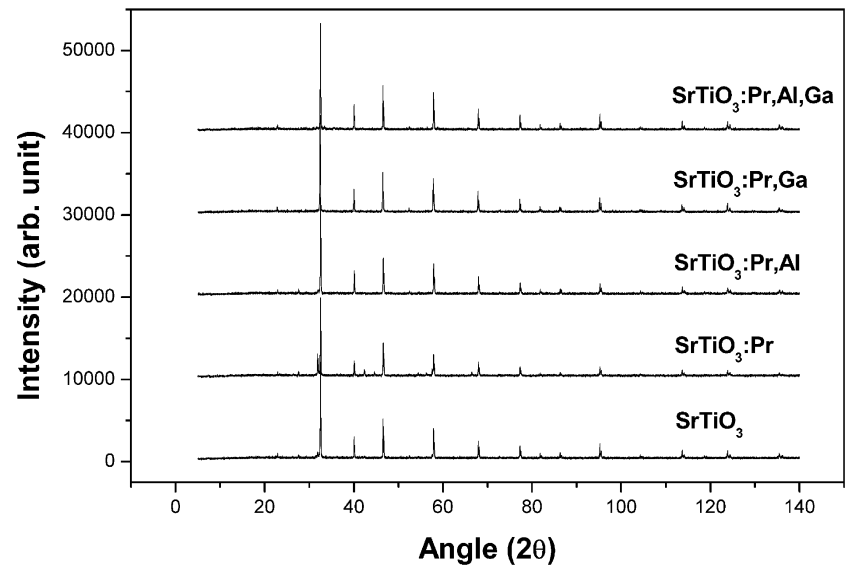

FIG. 1. Powder XRD patterns.

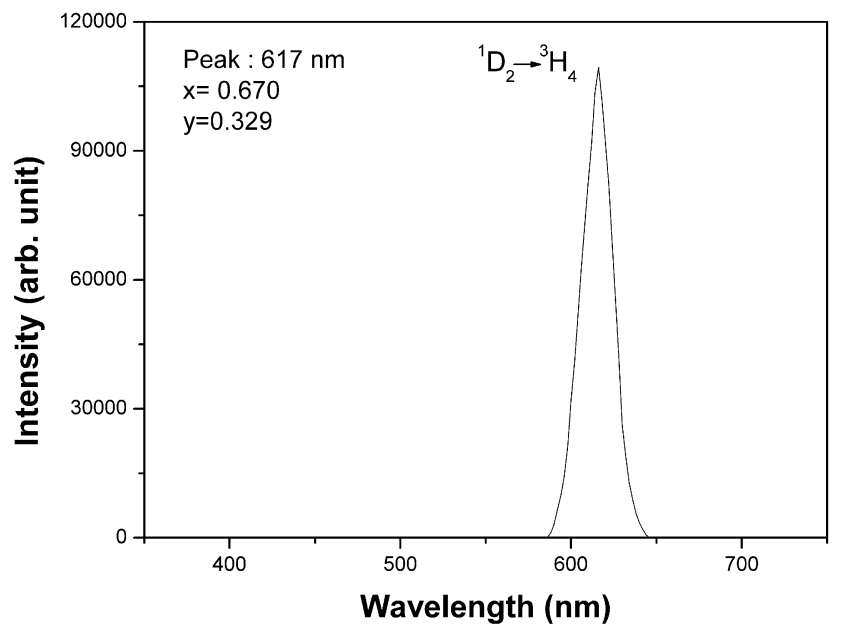

FIG. 2. Cathodoluminescence spectrum of $\mathrm{SrTiO}_{3}: \mathrm{Pr}, \mathrm{Al}, \mathrm{Ga}$ phosphors.

used a thermionic electron gun (Kimball Physics, Wilton, NH, FRA-2 $\times 1-4347$ B). The electron beam conditions during irradiation for the preparation of degraded samples were the electron acceleration voltage of 1000 $\mathrm{V}$, the excitation-current density of $157.9 \mu \mathrm{A} / \mathrm{cm}^{2}$, and the vacuum level of $3 \times 10^{-6}$ Torr. We noted that all the degraded area was visibly darker than the green areas of phosphors, and this made the alignment of subsequent measurements much easier.

\section{X-ray photoelectron spectroscopy}

X-ray photoelectron spectroscopy (XPS) was performed using a Physical Electronics (Kanagawa-ken, Japan) 5800 ESCA system equipped with a dual x-ray anode ( $\mathrm{Mg}$ and $\mathrm{Al})$. Data were obtained with $\mathrm{Al} \mathrm{K}_{\alpha}$ radiation $(1486.6 \mathrm{eV})$ at $300 \mathrm{~W}(15 \mathrm{keV}, 20 \mathrm{~mA})$. Survey scans were collected over the range $0-1100 \mathrm{eV}$ with $187.85 \mathrm{eV}$ pass energy detection. With $23.5 \mathrm{eV}$ pass energy detection, close-up scans were collected on the peaks of interest for the different elements. A base pressure of $10^{-9}$ Torr was maintained during the experiments.

\section{RESULTS AND DISCUSSION}

Figure 3 shows the degradation behavior of the $1 \mathrm{keV}$ CL measured on several low voltage oxide phosphors for electron doses up to $0.35 \mathrm{C} / \mathrm{cm}^{2}$. As shown in this figure, the most serious degradation of low voltage CL was observed in the STO phosphor among the analyzed above. It has been reported that the rapid degradation of CL of STO phosphor may be attributed to the larger amounts of carbon adsorption by electron-stimulated chemical reactions (ESCR) on the surface of the phosphor than the other phosphors under the same condition. ${ }^{12}$ Seager et al. demonstrated that extended electron-beam exposure produces a contaminating overlayer on phosphors, and this layer is graphitic in nature, arising from the electronbeam-stimulated conversion of hydrocarbons adsorbed from the vacuum ambient. ${ }^{14}$ Also, it has been known that the presence of this carbon overlayer adsorbed on the phosphor surface during electron irradiation may play a dominant role [for example, (i) absorption of the luminescence by the carbon overlayer, (ii) attenuation of the energy of the incoming electron beam, and (iii) alterations of phosphor charging behavior caused by the effect of the overlayer on the emission of secondary electrons, etc.]. This dominant role may be large enough to explain the rapid degradation of the CL process. However, in spite of such a serious effect of carbon adsorption on the phosphor surface, there are many ambiguous corners about the understanding of degradation mechanism. Thus, in this study, the mechanisms for heavy adsorption of carbon on the surface of STO phosphor leading to the rapid degradation of CL process are proposed. In this study XPS analysis for STO prior to and after electron irradiation was performed to study the detailed changes characterizing the surface chemical reaction on the phosphors during low voltage electron irradiation. The characteristic results in Figs. 4(a)-4(d) show XPS spectra of the $\mathrm{C}, \mathrm{Sr}, \mathrm{Ti}$, and $\mathrm{O}$ present on the surface of STO phosphors, respectively, prior to and after electron irradiation.

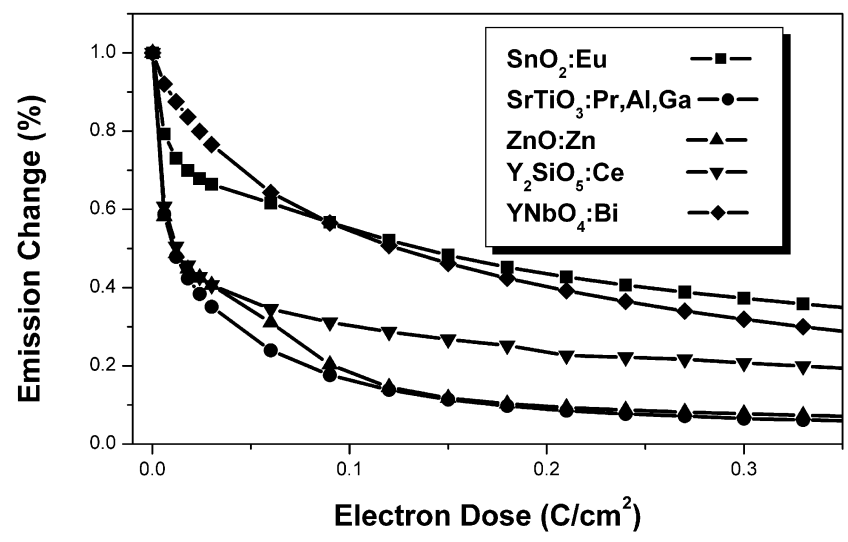

FIG. 3. Relative phosphor maintenance under low voltage electron irradiation. 


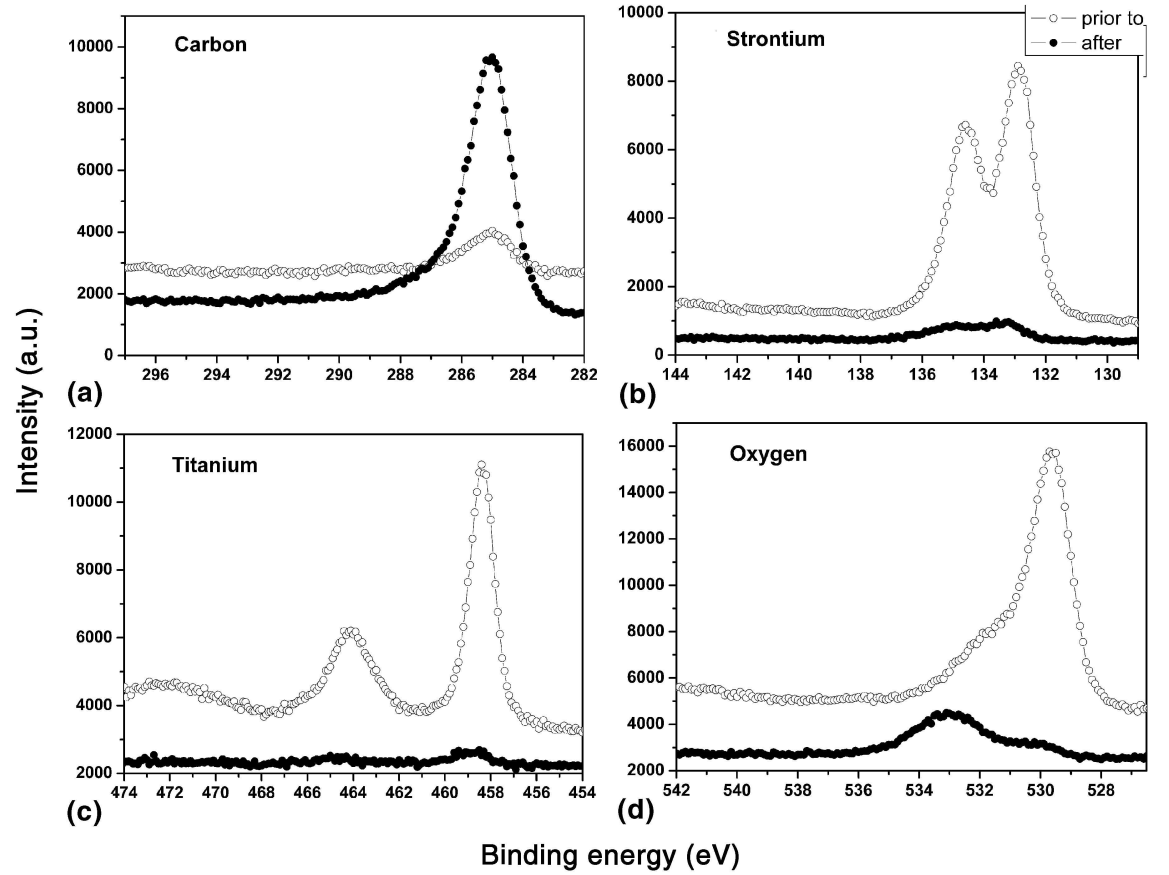

FIG. 4. XPS spectra for STO prior to and after electron irradiation: (a) carbon $1 s$, (b) strontium $3 d$, (c) titanium $2 p$, and (d) oxygen $1 s$.

As shown in Fig. 4(a), the XPS study showed that the amount of carbon absorbed increased during electron irradiation on the phosphor surface, which is consistent with previous results. ${ }^{12}$ However, the XPS signal of the other constituents except carbon for STO after electron irradiation decreased in overall intensity compared to that prior to electron irradiation. These results are consistent with the fact that the corresponding $\mathrm{Sr}, \mathrm{Ti}$, and $\mathrm{O}$ signal depletion is a consequence of the adsorbed carbon overlayer on the analyzed phosphor surface. Shape and binding energies of XPS spectra of constituents for STO showed little variation between them prior to and after electron irradiation, except for the $\mathrm{O} 1 \mathrm{~s}$ spectra; an intense peak at $531.7 \mathrm{eV}$ in the $\mathrm{O} 1 s$ spectra is observed. So, to further investigate the detailed changes of $\mathrm{O}$ signal for STO phosphors, XPS analysis of STO phosphor samples with a function of exposure time of extended electron beam was performed, shown in Fig. 5. In the case of the STO phosphors prior to electron irradiation, the binding energies of the $\mathrm{O} 1 \mathrm{~s}$ peaks were consistent with those reported for oxygen of STO. ${ }^{15}$ In Fig. 5(a), the main peak is seen at $529.5 \mathrm{eV}$ with a second peak at $531.2 \mathrm{eV}$. The main $\mathrm{O} 1 \mathrm{~s}$ peak at a binding energy of $529.5 \mathrm{eV}$ is ascribed to titanate oxygen. Sayers and Armstrong reported a main oxygen peak at $530.1 \mathrm{eV}$ for a STO single crystal, and this is in good agreement with the data shown in Fig. 5. ${ }^{16}$ The second oxygen peak seen at a binding energy of $531.2 \mathrm{eV}$, about $1.7 \mathrm{eV}$ higher than the titanate oxygen peak, is ascribed to the contribution of hydroxyl species. ${ }^{17}$ Also, it has been reported that this hydroxyl peak for STO comes from adsorption of

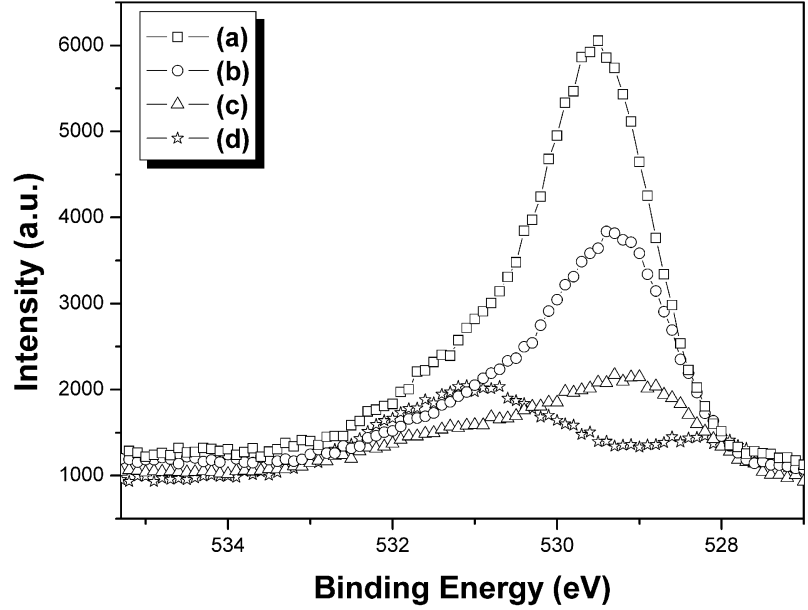

FIG. 5. Oxygen $1 s$ spectra for STO with a function of exposure time of extended electron beam: (a) $0 \mathrm{~s}$, (b) $500 \mathrm{~s}$, (c) $2000 \mathrm{~s}$, and (d) $3000 \mathrm{~s}$.

dissociative water molecules and is seen in all $\mathrm{O} 1 \mathrm{~s}$ spectra of STO ${ }^{15}$ However, as shown in Figs. 5(a)-5(d), with the increases of electron exposure time, the shape and binding energies of $\mathrm{O} 1 \mathrm{~s}$ spectra are significantly changed. The titanate oxygen peak at $529.5 \mathrm{eV}$ decreased steadily due to the addition of the carbon overlayer on the phosphor surface previous referred to, as shown in Figs. 4(b)-4(d). However, the intensity of hydroxyl peak has increased steadily and its peak has exhibited a small shift to a binding energy of $531.1 \mathrm{eV}$. As in the case of $\mathrm{TiO}_{2}$ surfaces, the interactions of water with STO surfaces have also attracted considerable attention due to the discovery of the photocatalytic properties of STO. ${ }^{18,19}$ 
Many researchers have applied photoemission spectroscopies and electron energy loss spectroscopy to study the adsoption and dissociation of water on STO. ${ }^{20-24}$ In particular, it has been reported that dissociative adsorption was observed for water only on reduced STO surfaces. ${ }^{18}$ In other words, water was found to be adsorbed in the form of molecule on stoichiometric STO surfaces, but dissociatively on reduced STO surfaces. Consequently, based on the XPS results shown in Fig. 5, it is understood that the increase of the hydroxyl peak in STO phosphors after electron irradiation is responsible for the oxygen loss in STO oxides in the perovskite structure. In practice, in the case of an oxide phosphor, the reduction in brightness appears to result from the reduction of the oxide due to its reaction with electron beam, apparently resulting in enhanced nonradiative surface electron-hole pair recombination. ${ }^{25}$ Also, the shift of hydroxyl peak is thought to be probably due to the weaker interaction between the titanate oxygen and the hydroxyl peaks because of a reduction of oxygen content. From the discovery of photoinduced water splitting on $\mathrm{TiO}_{2}$ electrodes in $1972, \mathrm{TiO}_{2}$ has been widely studied because of its potential industrial applications. ${ }^{26-28}$ When ultraviolet (UV) light is illuminated on it, electron and hole pairs are generated and they reduce and oxidize adsorbates on the surface, respectively, producing radical species such as $\mathrm{OH} \cdot$ and $\mathrm{HO}_{2}{ }^{-}$. These radicals decompose most organic compounds; thus, much research has been conducted on $\mathrm{TiO}_{2}$ with intention of its application to water and air purification. ${ }^{29,30}$ In addition to the $\mathrm{TiO}_{2}$, STO is known as an efficient photocatalyst for the production of gaseous hydrogen from water and for the decomposition of various organic compounds. ${ }^{31,32}$ Moreover the electronic structure of STO resembles that of $\mathrm{TiO}_{2}$, i.e., valence and conduction bands of these metal oxides consist mainly of O $2 p$-like orbitals and Ti $3 d$-like orbitals, respectively, with band gaps of about $3 \mathrm{eV}$. It is also reported that the STO surface is composed of Ti-O, and no $\mathrm{Sr}^{2+}$ ions are exposed. ${ }^{33}$ The present results indicate that the extended electron-beam exposure at low voltage and high current creates reduced STO on the STO surface, resulting from the dissociative adsorption of $\mathrm{H}_{2} \mathrm{O}$ contained in vacuum ambient. As a result of the dissociative adsorption of $\mathrm{H}_{2} \mathrm{O}$, STO produce various radicals such as $\mathrm{OH}$. and $\mathrm{HO}_{2}$, respectively, which decompose organic compounds chemisorbed on the surface in vacuum ambient eventually. Consequently, organic compounds chemisorbed on the surface of STO from the residual gases in vacuum ambient are decomposed, and deposited as a form of carbon-species on the STO surface. However, this process did not occur in the presence of background oxygen. Measurements performed by using residual gas analyzer proved that the oxygen components are not observed in vacuum ambient (not shown). Similarly, this phenomenon is observed in the case of $\mathrm{TiO}_{2}{ }^{34}$
Consequently, it is understood that this mechanism may attribute to the large amounts of carbon adsorption by ESCR on the surface of the STO phosphor, resulting in a rapid degradation in the phosphor performance.

\section{CONCLUSION}

We present basic experimental results on the characteristic changes present in the STO phosphors during low voltage electron irradiation. The amount of carbon adsorbed on the phosphor surface increased steadily with electron doses, resulting in a serious degradation in the phosphor performance, especially at low voltages. Also, based on the XPS results, we found that the oxygen is reduced from the STO phosphor under prolonged electron irradiation, which probably leads to the dissociative adsorption of $\mathrm{H}_{2} \mathrm{O}$ on the phosphor surface. The hydroxyl species produced by such dissociative adsorption of $\mathrm{H}_{2} \mathrm{O}$ strongly decompose organic compounds chemisorbed on the surface from vacuum ambient used in display devices into carbon species due to the photocatalytic properties of STO. Consequently, it is very likely that this mechanism attributes to the larger amounts of carbon adsorption by ESCR on the STO phosphor surface than other phosphors, resulting in a rapid degradation in the phosphor performance.

\section{REFERENCES}

1. L. Ozawa: Application of Cathodoluminescence to Display Devices (Kodansha, Tokyo, Japan, 1994).

2. T. Justel and H. Nikol: Optimization of luminescent materials for plasma display panels. Adv. Mater. 12, 527 (2000).

3. D.B.M. Klaassen and D.M. de Leeuw: Degradation of phosphors under cathode-ray excitation. J. Lumin. 37, 21 (1987).

4. S. Shionoya and W.M. Yen: Phosphor Handbook (CRC Press, Boca Raton, FL, 1999).

5. H.C. Swart, T.A. Trottier, J.S. Sebastian, S.L. Jones, and P.H. Holloway: Degradation of zinc sulfide phosphors under electron bombardment. J. Vac. Sci. Technol. A 14, 1697 (1996).

6. S. Itoh, T. Kimizuka, and T. Tonegawa: Degradation mechanism for low voltage cathodoluminescence of sulfide phosphors. J. Electrochem. Soc. 136, 1819 (1989).

7. S. Itoh, M. Yokoyama, and K. Morimoto: Poisonous gas effects on the emission of oxide-coated cathodes. J. Vac. Sci. Technol. A 5, 3430 (1987)

8. M. Yokoyama and S. Yang: Red $\mathrm{SrTiO}_{3}: \mathrm{Pr}, \mathrm{Al}$ phosphor as potential field emission display material. J. Vac. Sci. Technol. A 18, $2472(2000)$.

9. S. Itoh, H. Toki, K. Tamura, and F. Kataoka: A new red-emitting phosphor $\mathrm{SrTiO}_{3}: \mathrm{Pr}^{3+}$, for low-voltage electron excitation. Jpn. J. Appl. Phys. Part I 38, 6387 (1999).

10. S. Okamoto, H. Kobayashi, and H. Yamamoto: Enhancement of characteristic red emission from $\mathrm{SrTiO}_{3}: \mathrm{Pr}^{3+}$ by $\mathrm{Al}$ addition. J. Appl. Phys. 86, 5594 (1999)

11. Tamura Kiyoshi and Toki Hitoshi: Fluorescent Material and Display Tube. Japanese Patent No.10-273658 (1998).

12. J.Y. Kim, Y.C. You, D.Y. Jeon, I. Yu, and H.-G. Yang: A study on the degradation of cathodoluminescence of $\mathrm{SrTiO}_{3}: \mathrm{Pr}, \mathrm{Al}, \mathrm{Ga}$ 
phosphors tailored for low voltage display applications. $\underline{\text { J. Elec- }}$ trochem. Soc. 149, H44 (2002).

13. Y.C. You, J.Y. Kim, D.Y. Jeon, K.C. Park, S.H. Lee, and I. Yu: XPS observations of deterioration of $\mathrm{SrTiO}_{3}$ : $\mathrm{Pr}, \mathrm{Al}$, GA phosphor by low voltage electron irradiation, in The 2nd International Display Manufacturing Conference \& Exhibition, Seoul, Korea, (2002), p. 281.

14. C.H. Seager, D.R. Tallant, and W.L. Warren: Cathodoluminescence, reflectivity changes, and accumulation of graphitic carbon during electron beam aging of phosphors. J. Appl. Phys. 82, 4515 (1997).

15. P.V. Nagarkar, P.C. Searson, and F.D. Gealy: Effect of surface treatment on $\mathrm{SrTiO}_{3}$ : An x-ray photoelectron spectroscopic study. J. Appl. Phys. 69, 459 (1991).

16. C.N. Sayers and N.R. Armstrong: X-ray photoelectron spectroscopy of $\mathrm{TiO}_{2}$ and other titanate electrodes and various standard titanium oxide materials: Surface compositional changes of the $\mathrm{TiO}_{2}$ electrode during photoelectrolysis. Surf. Sci. 77, 301 (1978).

17. M.L. Knotek: Characterization of hydrogen species on metal oxide surfaces by electron-stimulated desorption: $\mathrm{TiO}_{2}$ and $\mathrm{SrTiO}_{3}$. Surf. Sci. 101, 334 (1980).

18. L-Q. Wang, K.F. Ferris, and G.S. Herman: Interactions of $\mathrm{H}_{2} \mathrm{O}$ with $\mathrm{SrTiO}_{3}$ (100) surfaces. J. Vac. Sci. Technol. A 20, 239 (2002).

19. M.S. Wrington, A.B. Ellis, P.T. Wolcznski, D.L. Morse, H.B. Abrahamson, and D.S. Gin: Strontium titanate photoelectrodes. Efficient photoassisted electrolysis of water at zero applied potential. J. Am. Chem. Soc. 98, 2774 (1976).

20. P.A. Cox, R.G. Egdell, and P.D. Naylor: HREELS studies of adsorbates on polar solids: Water on $\mathrm{SrTiO}_{3}$ (100). J. Electron. Spectrosc. Relat. Phenom. 29, 247 (1983).

21. V.E. Henrich, G. Dresselhaus, and H.J. Zeiger: Chemisorbed phases of $\mathrm{H}_{2} \mathrm{O}$ on $\mathrm{TiO}_{2}$ and $\mathrm{SrTiO}_{3}$. Solid State Commun. 24, 623 (1977).

22. C. Webb and M. Lichtensteiger: UPS/XPS study of reactive and non-reactive $\mathrm{SrTiO}_{3}(100)$ surfaces: Adsorption of $\mathrm{H}_{2} \mathrm{O}$. Surf. Sci. 107, L345 (1981).
23. S. Ferrer and G.A. Somorjai: Isotope exchange studies of the oxidation and reduction of $\mathrm{SrTiO}_{3}$ single crystal surfaces by water and hydrogen. Surf. Sci. 97, L304 (1980).

24. W.J. Lo and G.A. Somorjai: Temperature-dependent surface structure, composition, and electronic properties of the clean $\mathrm{Sr}$ $\mathrm{TiO}_{3}(111)$ crystal face: Low-energy-electron diffraction, Augerelectron spectroscopy, electron energy loss, and ultravioletphotoelectron spectroscopy studies. Phys. Rev. B 17, 4942 (1978).

25. P.R. Schwoebel, E.M. Pearson, K-H. Lau, D.H. Lowe, and A. Sanjuro: Lifetime extension of cathodoluminescent $\mathrm{P}-1$ phosphor. Electrochem. Solid-State Lett. 1, 102 (1998).

26. A. Fujishima and K. Honda: Electrochemical photocatalysis of water at a semiconductor electrode. Nature 238, 37 (1972).

27. M.R. Hoffmann, S.T. Martin, W. Choi, and D.W. Bahnemann: Environmental applications of semiconductor photocatalysis. Chem. Rev. 95, 69 (1995).

28. A. Heller: Chemistry and applications of photocatalytic oxidation of thin organic films. Acc. Chem. Res. 28, 503 (1995).

29. Y. Ohko, K. Hashimoto, and A. Fujishima: Kinetics of photocatalytic reactions under extremely low-intensity UV illumination on titanium dioxide thin films. J. Phys. Chem. A 101, 8057 (1997).

30. A. Mills and S.L. Hunte: An overview of semiconductor photocatalysis. J. Photochem. Photobiol. A: Chem. 108, 1 (1997).

31. J.G. Marroides, J.A. Kafalas, and D.F. Kolisar: Photoelectrolysis of water in cells with $\mathrm{SrTiO}_{3}$ anodes. Appl. Phys. Lett. 28, 241 $\underline{(1976)}$.

32. M.S. Wrighton, P.T. Wolczanski, and A.B. Ellis: Photoelectrolysis of water by irradiation of platinized n-type semiconducting metal oxide. J. Solid State Chem. 22, 17 (1977).

33. M. Miyauchi, A. Nakajima, A. Fujishima, K. Hashimoto, and T. Watanabe: Photoinduced surface reactions on $\mathrm{TiO}_{2}$ and $\mathrm{SrTiO}_{3}$ films: Photocatalytic oxidation and photoinduced hydrophilicity. Chem. Mater. 12, 3 (2000).

34. A.J.M. Mens and O.L.J. Gijzeman: AES study of electron beam induced damage on $\mathrm{TiO}_{2}$ surfaces. Appl. Surf. Sci. 99, 133 (1996). 